\title{
Connaissance locale des pratiques de la transhumance dans la zone soudano-sahélienne du Mali
}

\author{
Clarisse Umutoni ${ }^{1 *}$ Augustine Abioye Ayantunde ${ }^{2}$ \\ Germain Jérôme Sawadogo ${ }^{3}$
}

Mots-clés

Transhumance, communauté locale, système agropastoral, conflit foncier, élevage, Mali

Submitted: 5 December 2015 Accepted: 26 September 2016 Published: 26 October 2016

\section{Résumé}

Les études sur la transhumance ont souvent ciblé les pasteurs - notamment peuls - des zones sahéliennes. Pour en donner une autre perspective, la présente étude a été centrée sur les pratiques de la transhumance dans la zone soudano-sahélienne du Mali en intégrant la perception des agriculteurs et des pasteurs sédentaires concernés par la transhumance dans les zones d'accueil. Les scénarios envisageables pour le futur des systèmes pastoraux ont aussi été analysés. Les données ont été collectées à travers des groupes de discussion et des enquêtes individuelles. Les résultats ont montré que la transhumance n'était plus le seul fait des populations pastorales peules mais concernait diverses ethnies. Plus de $70 \%$ des agriculteurs, des pasteurs sédentaires et des pasteurs transhumants interviewés dans les deux sites de l'étude ont rapporté que les avantages de la transhumance pour les communautés d'accueil en termes de dépôt de fumier dans les champs des cultures décroissaient. Quand bien même la majorité des répondants ont rapporté une augmentation des pratiques de la transhumance au cours des trente dernières années, ils prévoyaient leur diminution dans les années à venir. L'augmentation de la compétition autour des ressources pastorales entre les membres des communautés d'accueil et les pasteurs transhumants dans le sud du Mali crée une situation d'impasse et des frustrations se manifestant par une méfiance mutuelle.

- Pour citer cet article : Umutoni C., Ayantunde A.A., Sawadogo G.J., 2016. Local knowledge of transhumance practices in the Sudano-Sahelian zone of Mali [in French]. Rev. Elev. Med. Vet. Pays Trop., 69 (2): 53-61

\section{INTRODUCTION}

Au cours des trente dernières années au Mali, la transhumance a connu des transformations importantes telles que l'augmentation du nombre d'animaux, l'augmentation de la distance parcourue et l'orientation des mouvements des animaux davantage tournée vers le Sud (Ayantunde et al., 2014 ; Bassett et Turner, 2007). Ces modifications ont occasionné un profond bouleversement des relations agriculture-élevage et une exacerbation de la compétition pour

\footnotetext{
1. International Livestock Research Institute, c/o Icrisat, BP 320, Bamako, Mali.
2. International Livestock Research Institute, Ouagadougou, Burkina Faso.

1. International Livestock Research Institute, c/o Icrisat, BP 320, Bamako, M
2. International Livestock Research Institute, Ouagadougou, Burkina Faso.

3. Département des sciences biologiques et productions animales, Université Cheikh Anta Diop, Dakar, Sénégal.

* Auteur pour la correspondance

Tél. : +22320709200; fax : +22320709201

Email : c.umutoni@cgiar.org
}

l'exploitation des ressources pastorales remettant en question la durabilité du système pastoral (Banoin et al., 1996).

La compétition pour l'exploitation des ressources pastorales (fourragères et eau) dans la zone soudano-sahélienne du Mali est ainsi le résultat de l'accroissement du nombre d'animaux transhumants dans cette zone pendant la saison sèche suite à la rareté des pâturages dans le Sahel ainsi que de l'augmentation du nombre d'animaux possédés par la communauté d'accueil. En effet, pour limiter les risques associés à la variabilité de la pluviométrie, les agriculteurs ont diversifié leurs activités en intégrant l'élevage du bétail dans leur système de production (Turner et al., 2014 ; Turner, 2000 cité par Ayantunde et al., 2014 ; Banoin et Jouve, 2000). Selon Moritz et al. (2009), la croissance démographique et la baisse des revenus agricoles due à la baisse de la fertilité des sols avec notamment la réduction des jachères seraient aussi d'autres éléments qui ont poussé les agriculteurs à diversifier leurs activités vers l'élevage. Parallèlement, cet accroissement démographique et la dégradation des terres cultivables dans les zones soudano-sahéliennes ont occasionné une expansion des zones de cultures au détriment des zones pastorales (ILRI, 2010). 
Il résulte de ces récentes tendances dans la zone soudano-sahélienne du Mali, une réduction et une marginalisation des espaces pastoraux, une augmentation de la charge animale et de la pression autour des ressources existantes, ainsi qu'une augmentation des tensions entre les éleveurs transhumants et les agriculteurs. Ces derniers se considèrent actuellement davantage comme des acteurs en compétition, alors qu'autrefois ils entretenaient des relations centrées sur la complémentarité. Ces changements ont affecté la perception des paysans sur les pratiques de la transhumance. Les études réalisées antérieurement ont surtout porté sur les pratiques de la transhumance dans le Sahel (Kiema et al., 2014 ; Turner et al., 2014 ; Ayantunde et al., 2000). De plus, elles se sont surtout intéressées aux éleveurs peuls et n'ont pas accordé beaucoup d'importance à la perception des autres acteurs dans les zones de transit et de destination des éleveurs transhumants venant du Sahel.

Cette étude prend en compte le point de vue de tous les acteurs concernés par la transhumance : agriculteurs ${ }^{4}$, pasteurs sédentaires ${ }^{5}$ et pasteurs transhumants ${ }^{6}$. Elle apporte aussi une valeur ajoutée à l'ensemble des connaissances sur la transhumance dans la zone subhumide où les études sur les pratiques de la transhumance sont limitées. L'objectif de cette étude a été d'examiner la perception des différents acteurs sur les pratiques et les tendances actuelles de la transhumance, et d'analyser les scénarios possibles sur l'avenir du pastoralisme dans la zone soudano-sahélienne du Mali.

\section{MATERIEL ET METHODES}

\section{Zone d'étude}

L'étude a été menée au sud du Mali dans la région de Sikasso et a concerné six communautés situées dans deux zones (figure 1) : dans la zone de Koutiala, Namposséla $\left(5,34^{\circ} \mathrm{O} ; 12,33^{\circ} \mathrm{N}\right)$, Sirakelé $\left(5,48^{\circ} \mathrm{O} ; 12,51^{\circ} \mathrm{N}\right)$ et Zanzoni $\left(5,57^{\circ} \mathrm{O} ; 12,61^{\circ} \mathrm{N}\right)$; et dans la zone de Bougouni, Diéba $\left(8^{\circ} \mathrm{O} ; 10,91^{\circ} \mathrm{N}\right)$, Sibilira $\left(7,76^{\circ} \mathrm{O} ; 11,44^{\circ} \mathrm{N}\right)$ et Yorobougoula $\left(7,91^{\circ} \mathrm{O} ; 11,52^{\circ} \mathrm{N}\right)$. Le climat qui règne dans la zone d'étude est de type soudanien, caractérisé par une alternance de

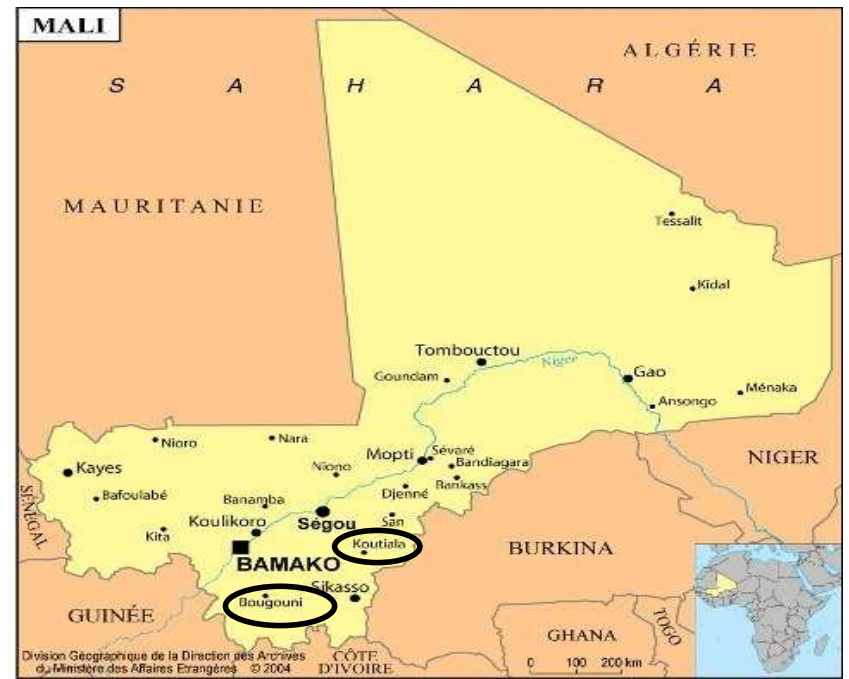

Figure 1 : localisation de la zone d'étude au Mali.

${ }^{4}$ Paysans autochtones du sud du Mali dont l'activité principale est l'agriculture mais qui ont intégré les activités d'élevage pour soutenir leurs activités agricoles et diversifier les revenus du ménage

${ }^{5}$ Paysans éleveurs qui se sont sédentarisés dans le sud du Mali

${ }^{6}$ Eleveurs pour lesquels a) l'élevage constitue l'activité principale et b) le système de production se caractérise par la mobilité saisonnière saison sèche et de pluie d'une durée moyenne de six mois. La pluviométrie annuelle varie respectivement entre 750 et $1100 \mathrm{~mm}$, et 900 et $1200 \mathrm{~mm}$ dans les zones de Koutiala et de Bougouni. L'économie des deux zones repose principalement sur les activités agrosylvopastorales. Cette région a connu des vagues de migrations venues du centre et du nord du pays après les années de sécheresse. Beaucoup de Dogons et de Peuls y ont trouvé des terres d'accueil favorables à leurs activités agricoles et pastorales (Dembélé, 2008). Les deux zones ont été sélectionnées par le projet Africa Rising car elles se situent dans les zones d'intervention prioritaires pour les activités de l'USAID au Mali. En outre, les deux zones sont très importantes pour les pratiques de la transhumance au Mali, et elles constituent les points de transit et/ou de destination des éleveurs transhumants.

\section{Collecte des données}

Les données ont été collectées en période de transhumance, de février à avril 2014, pour assurer l'inclusion du point de vue des éleveurs transhumants. Elles ont été recueillies à travers des groupes de discussion (Duggleby, 2005) et pendant une enquête transversale exhaustive (Creswell, 2014). Le cumul des entretiens individuels et collectifs était justifié par leur complémentarité. Leur combinaison a permis d'explorer en détail les opinions des paysans et les expériences spécifiques. Le tableau I donne un aperçu sur les méthodes de collecte des données.

\section{Groupes de discussion}

Les groupes de discussion ont été conduits sous forme d'entretien semi-structuré dans le but de répondre à la question suivante : quelles ont été les tendances des pratiques de la transhumance au cours des trente dernières années ? Des discussions ont ainsi eu lieu pour permettre une compréhension aussi complète que possible du sujet et pour recueillir les perceptions des populations concernées.

Ces groupes de discussion ont été organisés par communauté : trois dans la zone de Bougouni et trois dans celle de Koutiala. Pour faciliter les échanges, quinze personnes au plus et huit personnes au minimum ont été invitées pour constituer chaque groupe. Seuls les paysans résidant dans les communautés d'étude (agriculteurs et pasteurs sédentaires) ont été concernés. Par ailleurs, aucun groupe ethnique n'a été privilégié, le choix a été fait pour que les participants représentent une couche importante de la population visée. La connaissance pertinente du sujet, la volonté et la capacité de partager ces connaissances ont été d'autres critères qui ont orienté la sélection des participants. Les discussions ont été centrées sur l'évaluation des tendances des pratiques de la transhumance. Pour cela, une proposition de variables sur la transhumance a été présentée et il a été demandé aux participants si celles-ci avaient augmenté ou diminué au cours des trente dernières années. Ensuite, il leur a été demandé d'apprécier l'ampleur du changement (faible, modéré, élevé, très élevé). Les détails ont été collectés à travers les discussions menées autour de chaque variable (thème). Les thèmes abordés ont été la mobilité du bétail dans le terroir du village, la distance parcourue lors de la transhumance, l'accès aux pâturages et à l'eau par les troupeaux transhumants, la relation des éleveurs pastoraux avec la communauté d'accueil, et la fréquence des conflits entre les agriculteurs et les éleveurs transhumants.

\section{Enquêtes individuelles}

Les enquêtes individuelles ont été menées pour répondre aux questions suivantes : a) quelle est la perception des acteurs concernés par la transhumance des pratiques de la transhumance ? et b) comment ces acteurs perçoivent-ils les pratiques de la transhumance dans les années à venir? Les enquêtes ont été conduites auprès de 177 paysans. Les entretiens semi-structurés ont été centrés sur trois groupes concernés par la transhumance dans chaque communauté : les agriculteurs, les pasteurs sédentaires et les pasteurs transhumants. Il est à 
Tableau I

Méthodes de collecte de données auprès des paysans de deux zones au sud du Mali

\begin{tabular}{|c|c|c|c|c|c|c|}
\hline \multirow[t]{2}{*}{ Méthode } & \multirow[t]{2}{*}{ Zone } & \multicolumn{4}{|c|}{ Nombre des paysans impliqués } & \multirow[t]{2}{*}{ Restitution } \\
\hline & & Homme & Femme & Total & Groupe & \\
\hline \multirow[t]{2}{*}{ Groupe de discussion } & Bougouni & 29 & 10 & 39 & $\begin{array}{c}\text { Agriculteur } \\
\text { et pasteur résidant }\end{array}$ & \multirow[t]{2}{*}{$\begin{array}{l}\text { Perceptions des paysans sur } \\
\text { les tendances actuelles de } \\
\text { la transhumance dans } \\
\text { la communauté au cours } \\
\text { des } 30 \text { dernières années }\end{array}$} \\
\hline & Koutiala & 31 & 14 & 45 & & \\
\hline \multirow[t]{6}{*}{ Enquête individuelle } & \multirow[t]{3}{*}{ Bougouni } & 37 & 8 & 45 & Agriculteur & \multirow{6}{*}{$\begin{array}{l}\text { Perceptions concernant } \\
\text { les pratiques actuelles } \\
\text { de la transhumance } \\
\text { Perception sur l'avenir } \\
\text { de la transhumance }\end{array}$} \\
\hline & & 13 & 4 & 17 & Pasteur résidant & \\
\hline & & 24 & 0 & 24 & Pasteur transhumant & \\
\hline & \multirow[t]{3}{*}{ Koutiala } & 53 & 12 & 65 & Agriculteur & \\
\hline & & 16 & 2 & 18 & Pasteur résidant & \\
\hline & & 8 & 0 & 8 & Pasteur transhumant & \\
\hline
\end{tabular}

préciser que dans les deux zones (Bougouni et Koutiala), davantage d'agriculteurs ont été interviewés en raison de leur nombre élevé par rapport aux autres groupes. Le choix des personnes à interroger dans chaque groupe a été fait au hasard, sauf dans les cas où la taille de l'échantillon total était trop petite, comme pour le groupe des pasteurs transhumants ; ainsi tous les éleveurs transhumants rencontrés lors des enquêtes ont été interviewés.

Pour évaluer la perception des paysans sur les pratiques de la transhumance, une échelle de Likert a été utilisée pour classer les opinions. C'est une échelle de jugement avec laquelle la personne interrogée exprime son degré d'accord ou de désaccord vis-à-vis d'une affirmation. Une note est attribuée à chaque réponse proposée. Dans notre étude, trois types de réponses ont été proposées : 1 = d'accord ; 2 = neutre $; 3=$ en désaccord.

\section{Analyses statistiques}

Une analyse descriptive a été menée sur les perceptions des paysans vis-à-vis des tendances des pratiques de la transhumance. Les citations de propos ont été ajoutées dans certains cas pour la mise en scène des perceptions des répondants. Le logiciel SPSS version $20 \mathrm{a}$ été utilisé pour certaines analyses descriptives.

\section{RESULTATS}

\section{Tendances de la transhumance au cours des trente dernières années}

Les informations collectées à travers les groupes de discussion conduits dans les zones de Bougouni et de Koutiala ont montré que les pratiques de la transhumance dans la région soudano-sahélienne du Mali au cours des trente dernières années ont fait face à quelques changements dont la nature et l'importance ont varié selon la zone d'étude. Il y a eu une augmentation du nombre de troupeaux partant en transhumance. L'ampleur attribuée à cette augmentation par les répondants dans les discussions de groupe a été très élevée à Bougouni et élevée à Koutiala. Les participants ont perçu cette augmentation comme le résultat de la réduction de la surface des pâturages dans beaucoup de lieux. « Il y a plus d'animaux et moins de ressources, il faut alors multiplier les déplacements des animaux pour couvrir leurs besoins », ont expliqué les participants. Selon les paysans agriculteurs et pasteurs sédentaires dans les zones d'accueil, l'accès aux pâturages et à l'eau pour les troupeaux transhumants a très fortement diminué dans les deux zones au cours des trente dernières années. L'appropriation des animaux par les agriculteurs des communautés d'accueil a été perçue comme un élément qui a contribué à la réduction de l'accès au pâturage et à l'eau pour les troupeaux transhumants. « Puisque nous avons beaucoup d'animaux, nous privilégions d'abord nos animaux. De plus, rares sont maintenant les éleveurs transhumants qui ont accès aux résidus de récolte car nous collectons la plupart de ces résidus pour les distribuer à nos propres animaux », ont rapporté les agriculteurs lors de discussions de groupe.

Les paysans pensent qu'aujourd'hui, la descente des éleveurs transhumants vers le sud du Mali est précoce comparée aux trente années passés : «De temps en temps les éleveurs transhumants arrivent avant même les périodes de récoltes ». Globalement, selon les participants des groupes de discussion, aujourd'hui on peut distinguer deux types de flux du bétail qui se distinguent en fonction de la période d'arrivée des transhumants dans les zones de transit et/ou de destination. Il s'agit, d'une part, des vagues d'animaux transhumants arrivant dans les zones de transit ou d'accueil avant, pendant ou juste après les récoltes (entre les mois d'octobre et de janvier). « La majorité des animaux transhumants arrivent durant cette période », ont spécifié les paysans, ajoutant que la présence des animaux transhumants durant cette période était à l'origine d'énormes dégâts dans leurs champs. D'autre part, il y a les vagues d'animaux transhumants arrivant un peu en retard dans les zones de transit ou d'accueil quand les champs sont complètement dégagés (entre février et mai). A cette période, les transhumants avaient l'accès libre aux résidus de récolte restant dans les champs après que les agriculteurs aient ramassé la quantité nécessaire de résidus pour leurs propres animaux. Ces deux types de transhumants retournaient chez eux à l'approche de l'hivernage ou dès le début de l'hivernage (mai-juin).

En résumé, les mouvements des animaux dans les territoires de Bougouni et de Koutiala s'étalaient d'octobre à mai avec des pics observés en novembre ou décembre selon les répondants. A Bougouni, un troisième type de transhumants circulant dans cette zone pendant les périodes de cultures a été constaté ces dernières années. D'après les paysans, ces transhumants ne retournaient jamais chez eux, ils étaient présents tout au long de l'année et se déplaçaient d'une zone à l'autre dans le sud du Mali en fonction des périodes (période de culture, de récolte et d'après récoltes) mais aussi en fonction de la disponibilité de ressources pastorales. Selon les répondants, ils n'étaient pas nombreux. 
La réponse à la question sur la variabilité de la durée de séjour des transhumants dans les zones d'étude a révélé que cette durée a fortement diminué à Koutiala : " Aujourd'hui les transhumants ne font que passer ». Dans les enquêtes individuelles, la durée moyenne de présence des bergers transhumants à Koutiala a été de 2,33 $\pm 0,52$ jours. « La disponibilité des pâturages est devenue un problème, la plupart des zones qui étaient dans le passé dédiées aux activités pastorales ont été transformées en zones de culture », ont expliqué les paysans, ce qui a réduit le temps de séjour des transhumants à Koutiala.

Contrairement à Koutiala, la durée de séjour des transhumants dans la zone de Bougouni a très fortement augmenté avec une moyenne de 60,82 $\pm 6,36$ jours. La disponibilité en ressources pastorales dans cette zone constituant un facteur favorable. De plus, cette zone est limitrophe avec la Côte d'Ivoire et les transhumants qui, dans le passé, la traversaient pour se rendre dans ce pays ont été obligés de s'y arrêter en raison des problèmes politiques ivoiriens.

Les trente dernières années ont été caractérisées par des changements dans les relations entre les éleveurs pastoraux et les agriculteurs des communautés d'accueil ou de transit. Les paysans ont perçu ce changement dans les relations entre les éleveurs transhumants et les communautés d'accueil en termes de diminution des échanges et des contacts, et d'augmentation des conflits.

A Bougouni, les agriculteurs et les pasteurs résidants ont rapporté que leurs relations avec les éleveurs transhumants sont devenues de plus en plus tendues. Les principaux facteurs cités comme étant à l'origine de ce changement étaient les conflits et les dégâts causés par les animaux transhumants. Selon les dires des paysans, les comportements des pasteurs transhumants ont complètement changé : « Ils sont devenus violents, ils n'ont plus de respect ni peur de rien, ce qui importe c'est l'alimentation de leurs animaux ». Les paysans ont attribué la forte augmentation des conflits à une compétition accrue pour les ressources pastorales dans cette zone de transit ou de destination pour un grand nombre de transhumants venant de plusieurs régions et avec des mentalités différentes.

Selon les répondants la fréquence des conflits a moyennement diminué à Koutiala pour les raisons suivantes : «Le paysan est devenu ouvert, le dialogue entre les gens et l'esprit d'écoute ont été mis au centre de toutes nos relations. De plus, presque tous les paysans combinent actuellement l'agriculture et l'élevage, il est devenu alors facile de nous tolérer surtout en cas de dégâts causés par les animaux dans les champs ». Les paysans optaient pour un règlement à l'amiable plutôt que conflictuel. Un autre élément majeur mentionné comme ayant contribué à la réduction des conflits dans cette zone était le raccourcissement de la durée de séjour des transhumants.

\section{Perception paysanne sur les pratiques de la transhumance}

La perception des paysans sur les pratiques de la transhumance à Bougouni et à Koutiala est présentée dans le tableau II. Plus de $90 \%$ des paysans concernés, c'est-à-dire les agriculteurs, les pasteurs sédentaires et les éleveurs transhumants dans les deux zones, ont reconnu que la transhumance était caractérisée par un long déplacement saisonnier des animaux toujours à la recherche de pâturages et que dernièrement cette mobilité était beaucoup plus orientée vers le Sud. A Bougouni, seuls les éleveurs transhumants ont indiqué que la transhumance constituait un moyen pour maintenir la résilience écologique des écosystèmes des zones arides et assurer la productivité de l'élevage. En revanche, à Koutiala, tous les acteurs ont affirmé cela.

A Bougouni, la grande majorité des paysans, soit $58 \%$ des agriculteurs, $65 \%$ des pasteurs sédentaires et $79 \%$ des éleveurs transhumants, était d'accord sur le fait que la transhumance impliquait toujours de grands troupeaux en provenance de la zone sahélienne. Ils ont associé cela à la rareté des ressources fourragères et en eau dans cette zone mais aussi au grand nombre de troupeaux possédé par les éleveurs. En revanche à Koutiala, plus de $60 \%$ des paysans interviewés dans chaque catégorie des répondants ont été en désaccord avec cette affirmation. Ils ont déclaré que la transhumance était pratiquée par des troupeaux en provenance de tous les horizons : " Ils viennent de zones où il n'y a pas suffisamment de ressources pastorales ». Ils ont ajouté que tout paysan, qu'il soit du nord ou d'ailleurs, propriétaire de plus 20 têtes de bovins était obligé de déplacer ses animaux pendant la saison sèche pour couvrir leurs besoins alimentaires. « Même certains agriculteurs de la zone de Koutiala et des zones environnantes où les ressources pastorales commencent à se faire rares font la transhumance en allant vers l'extrême sud du pays ", ont déclaré certains interviewés. Ces agriculteurs partaient après les périodes de récolte, juste après avoir utilisé les résidus, et ils revenaient à l'approche de l'hivernage pour pouvoir participer aux travaux agricoles (de février à mai).

La majorité des acteurs concernés par la transhumance dans les deux zones d'étude ont précisé que les Peuls n'étaient plus les seuls à pratiquer la transhumance. En effet, un résultat important de notre étude est que la transhumance est aujourd'hui pratiquée par une diversité de groupes ethniques (figure 2). Il n'y a pas eu de différence de perception entre les agriculteurs et les éleveurs transhumants. Bien que la quasi-totalité des répondants aient affirmé que les pasteurs peuls restaient les plus nombreux des éleveurs transhumants, ils ont constaté que d'autres groupes ethniques pratiquent la transhumance aujourd'hui. Les groupes ethniques les plus souvent rencontrés à Bougouni étaient les Bambara et les Soninkés, et à Koutiala les Miniankan et les Bella. Hormis les pasteurs transhumants enquêtés à Bougouni, la majorité des autres catégories de répondants à Bougouni et à Koutiala a mentionné que la pratique de la transhumance n'a pas diminué au cours des trente dernières années : «Au contraire, la pratique de la transhumance ne fait qu'augmenter d'une année à l'autre et prend une ampleur beaucoup plus élevée ». Néanmoins, une minorité a affirmé que la pratique de la transhumance a diminué au cours de cette période.

Plus de $80 \%$ des répondants toutes catégories confondues dans les deux zones ont confirmé qu'il était difficile de gérer les troupeaux transhumants en termes de contrôle des mouvements des animaux sur les territoires des zones de transit ou d'accueil et d'accès aux ressources pastorales. La perte et le vol d'animaux dus à l'arrivée massive des éleveurs transhumants dans les communautés d'accueil ou de transit ont été relatés par une grande proportion des agriculteurs et des pasteurs sédentaires enquêtés dans les deux zones. Cependant, plus de $75 \%$ des éleveurs transhumants n'ont pas été d'accord avec cette déclaration : «C'est le manque d'attention de la part des agriculteurs. Parfois leurs animaux peuvent même nous suivre sans qu'on s'en rende compte ». A Bougouni, 81 sur 86 paysans enquêtés ont affirmé que la présence de troupeaux transhumants conduisait souvent à des conflits, contre 56 sur 91 paysans enquêtés à Koutiala.

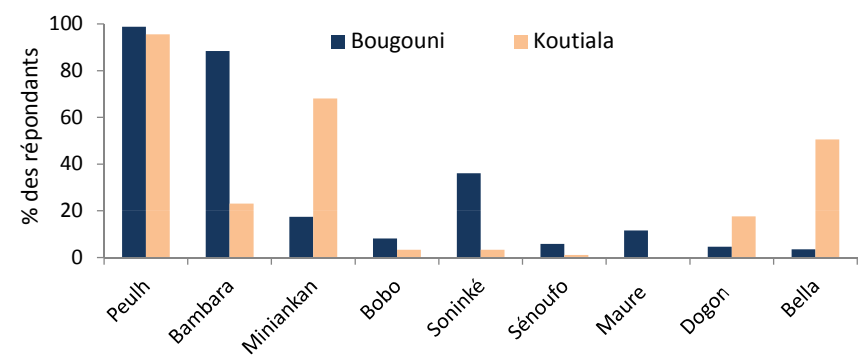

Figure 2 : groupes ethniques des éleveurs pratiquant la transhumance au sud du Mali selon la perception des paysans. 


\section{Tableau II}

Perception des paysans sur les pratiques de la transhumance (TR) dans deux zones du sud du Mali (\%)

\begin{tabular}{|c|c|c|c|c|c|c|}
\hline & \multicolumn{3}{|c|}{ Bougouni } & \multicolumn{3}{|c|}{ Koutiala } \\
\hline & Agriculteur & $\begin{array}{l}\text { Pasteur } \\
\text { sédentaire }\end{array}$ & $\begin{array}{c}\text { Pasteur } \\
\text { transhumant }\end{array}$ & Agriculteur & $\begin{array}{l}\text { Pasteur } \\
\text { sédentaire }\end{array}$ & $\begin{array}{c}\text { Pasteur } \\
\text { transhumant }\end{array}$ \\
\hline \multicolumn{7}{|c|}{ TR est caractérisée par un long déplacement saisonnier des animaux } \\
\hline D’accord & 100 & 100 & 100 & 100 & 100 & 87,5 \\
\hline Neutre & 0 & 0 & 0 & 0 & 0 & 0 \\
\hline En désaccord & 0 & 0 & 0 & 0 & 0 & 12,5 \\
\hline \multicolumn{7}{|c|}{ TR est pratiquée seulement par les groupes ethniques peuls } \\
\hline $\mathrm{D}^{\prime}$ accord & 6,7 & 11,8 & 4,2 & 6,2 & 0 & 0 \\
\hline Neutre & 0 & 11,8 & 0 & 4,6 & 0 & 0 \\
\hline En désaccord & 93,3 & 76,4 & 95,8 & 89,2 & 100 & 100 \\
\hline \multicolumn{7}{|c|}{ TR est essentielle pour maintenir la résilience écologique des écosystèmes des zones arides et assurer la productivité de l'élevage } \\
\hline D'accord & 22,2 & 5,9 & 75 & 93,8 & 94,4 & 100 \\
\hline Neutre & 73,3 & 88,2 & 25 & 4,6 & 0 & 0 \\
\hline En désaccord & 4,5 & 5,9 & 0 & 1,5 & 5,6 & 0 \\
\hline \multicolumn{7}{|c|}{ TR implique toujours de grands troupeaux en provenance de la zone sahélienne } \\
\hline D'accord & 57,8 & 64,7 & 79,2 & 15,4 & 22,2 & 12,5 \\
\hline Neutre & 4,4 & 0 & 8,3 & 6,2 & 16,7 & 25,0 \\
\hline En désaccord & 37,8 & 35,3 & 12,5 & 78,5 & 61,1 & 62,5 \\
\hline \multicolumn{7}{|c|}{ La pratique a diminué au cours des 30 dernières années } \\
\hline D'accord & 22,2 & 41,2 & 54,2 & 15,4 & 5,6 & 0 \\
\hline Neutre & 8,9 & 17,6 & 0 & 4,6 & 0 & 12,5 \\
\hline En désaccord & 68,9 & 41,2 & 45,8 & 80 & 94,4 & 87,5 \\
\hline \multicolumn{7}{|c|}{ Il est toujours difficile de gérer les troupeaux transhumants sur le territoire du village } \\
\hline D'accord & $95,6^{\circ}$ & 94,1 & 54,2 & 92,3 & 77,8 & 75 \\
\hline Neutre & 2,2 & 5,9 & 8,3 & 1,5 & 11,1 & 25 \\
\hline En désaccord & 2,2 & 0 & 37,5 & 6,2 & 11,1 & 0 \\
\hline \multicolumn{7}{|c|}{ La présence de troupeaux transhumants conduit souvent à des conflits } \\
\hline $\mathrm{D}^{\prime}$ accord & 93,3 & 100 & 91,7 & 61,5 & 66,6 & 37,5 \\
\hline Neutre & 2,2 & 0 & 8,3 & 3,1 & 16,7 & 25 \\
\hline En désaccord & 4,4 & 0 & 0 & 35,4 & 16,7 & 37,5 \\
\hline \multicolumn{7}{|c|}{ TR sera significativement réduite dans les 30 prochaines années } \\
\hline D'accord & 71,1 & 70,6 & 83,3 & 60 & 44,4 & 50 \\
\hline Neutre & 6,7 & 11,8 & 4,2 & 3,1 & 16,7 & 12,5 \\
\hline En désaccord & 22,2 & 17,6 & 12,5 & 36,9 & 38,9 & 37,5 \\
\hline \multicolumn{7}{|c|}{ TR augmente la compétition autour des ressources naturelles (pâturages, eau, résidus de récolte) dans la communauté } \\
\hline D’accord & 95,6 & 94,1 & 83,3 & 96,9 & 83,3 & 100 \\
\hline Neutre & 4,4 & 5,9 & 8,3 & 0 & 11,1 & 0 \\
\hline En désaccord & 0 & 0 & 8,4 & 3,1 & 5,6 & 0 \\
\hline \multicolumn{7}{|c|}{ Les avantages de TR en dépôt de fumier dans les champs de cultures pour la communauté d'accueil diminuent } \\
\hline D'accord & 86,7 & 88,2 & 91,7 & 100 & 77,8 & 100 \\
\hline Neutre & 2,2 & 0 & 0 & 0 & 11,1 & 0 \\
\hline En désaccord & 11,1 & 11,8 & 8,3 & 0 & 11,1 & 0 \\
\hline \multicolumn{7}{|c|}{ Les communautés d'accueil sont toujours amicales et coopérative avec les transhumants } \\
\hline $\mathrm{D}^{\prime}$ accord & 48,9 & 5,9 & 12,5 & 80 & 55,6 & 87,5 \\
\hline Neutre & 11,1 & 23,5 & 33,3 & 18,5 & 44,4 & 12,5 \\
\hline En désaccord & 40 & 70,6 & 54,2 & 1,5 & 0 & 0 \\
\hline \multicolumn{7}{|c|}{ La présence de troupeaux transhumants conduit toujours à la perte d'animaux pour les populations autochtones } \\
\hline D'accord & 86,7 & 88,2 & 16,7 & 69,2 & 55,6 & 12,5 \\
\hline Neutre & 6,7 & 5,9 & 4,2 & 6,2 & 16,7 & 0 \\
\hline En désaccord & 6,7 & 5,9 & 79,2 & 24,6 & 27,8 & 87,5 \\
\hline \multicolumn{7}{|c|}{ TR est une pratique démodée ou ancienne qui devrait être interdite } \\
\hline $\mathrm{D}^{\prime}$ accord & 77,8 & 70,6 & 29,2 & 20 & 5,6 & 0 \\
\hline Neutre & 2,2 & 17,6 & 4,2 & 21,5 & 33,3 & 12,5 \\
\hline En désaccord & 20 & 11,8 & 66,6 & 58,5 & 61,1 & 87,5 \\
\hline
\end{tabular}


Aussi bien les agriculteurs que les éleveurs transhumants ont partagé ce point de vue. La plupart des conflits enregistrés étaient liés aux dégâts causés au niveau des champs, d'autant que le passage était très difficile dans les zones ne disposant pas de schémas pastoraux. Néanmoins à Koutiala, 29 sur 91 interviewés ont déclaré que, bien que la gestion des animaux transhumants dans leurs zones fût difficile, elle ne conduisait pas souvent à des conflits.

Les agriculteurs et les pasteurs sédentaires et transhumants ont aussi mentionné que les avantages de la transhumance pour les communautés d'accueil en termes de dépôt de fumier dans les champs des cultures décroissaient. Ceci a été reconnu par plus de $70 \%$ de l'ensemble des interviewés. Selon eux, cette baisse du dépôt de fumier dans les champs était due, en premier lieu, à la réduction de la durée de séjour des transhumants dans les champs des agriculteurs. Cette réduction a été la conséquence du manque de ressources pastorales, surtout à Koutiala où les transhumants ne faisaient que passer et ne déposaient donc pas suffisamment de bouses dans les champs. En deuxième lieu, la plupart des agriculteurs sont devenus réticents à l'idée de laisser les animaux transhumants camper dans leurs champs car ils étaient convaincus que leur passage apportaient des mauvaises herbes. En dernier lieu, ils ont mentionné le manque de points d'eau pour l'abreuvement des animaux. D'après les transhumants, l'abreuvement des animaux était de plus en plus problématique et ils ne pouvaient pas rester dans les zones où il leur était difficile d'abreuver leurs troupeaux. En outre, plus de $80 \%$ des éleveurs transhumants enquêtés ont rapporté que les agriculteurs ramassaient presque la totalité des résidus après les récoltes et qu'il n'y avait ainsi aucune raison d'aller camper dans les champs des agriculteurs : «C'est du business, pas d'eau, pas de résidus, alors pas question de leur fournir du fumier ». Les transhumants préféraient camper dans la brousse plutôt que dans les champs, là ils étaient sûrs de trouver les aliments pour leurs animaux.

\section{Perception des paysans sur l'avenir des pratiques de la transhumance}

Pour comprendre la perception des paysans sur l'avenir des pratiques de la transhumance, il a été demandé aux acteurs concernés d'exprimer leurs prévisions sur cette question. A Bougouni, $71 \%$ des agriculteurs, $70 \%$ des pasteurs sédentaires et $83 \%$ des pasteurs transhumants ont affirmé que la transhumance serait significativement réduite dans les trente prochaines années, contre seulement et respectivement 60,44 et $50 \%$ de ces groupes interviewés à Koutiala.

\section{Perception des paysans sur la réduction des pratiques de la transhumance}

Les raisons données par les interviewés dans les deux zones pour la réduction des pratiques de la transhumance ont été regroupées en trois catégories décrites ci-après.

\section{Raisons climatiques et environnementales}

Selon $70 \%$ de la totalité des répondants dans les deux zones, la probabilité d'une réduction significative des pratiques de la transhumance vers la région du sud du Mali serait le résultat des perturbations climatiques et des dégradations environnementales. Leur argumentation a été fondée sur l'hypothèse que la persistance des perturbations climatiques allait conduire à une réduction considérable des ressources pastorales dans le sud et un peu partout dans le pays. Dans cette situation, il serait alors difficile aux éleveurs de couvrir les besoins de leurs animaux. Ainsi, les éleveurs seraient obligés de rester chez eux et de créer des conditions alternatives pour nourrir leur bétail sur place.

\section{Raisons liées aux relations sociales}

Plus de $90 \%$ des répondants agriculteurs, pasteurs sédentaires et transhumants à Bougouni contre $30 \%$ à Koutiala ont pointé l'aggravation des relations sociales, menant parfois à la violence, entre les transhumants et les membres des communautés d'accueil ou de transit, comme l'une des raisons de la probabilité d'une réduction significative des pratiques de la transhumance. Leurs critiques ont montré que la persistance de telles situations allait créer des frustrations au niveau des communautés d'accueil et qu'aucune communauté n'accepterait d'accueillir les transhumants, voire de les laisser passer dans la communauté. Dans ces conditions, les transhumants seraient bloqués et il leur serait difficile de maintenir leurs déplacements. Certains agriculteurs ont même fait des déclarations menaçantes : «Avec cette ampleur des conflits, si l'Etat ne trouve pas de solution adéquate, on saura quoi faire $»$.

\section{Raison de la gestion de troupeau des éleveurs transhumants}

« La gestion des animaux pour couvrir leurs besoins alimentaires devient de plus en plus difficile », ont déclaré plus de la moitié des interviewés toutes catégories confondues. Ils ont affirmé être conscients du déséquilibre entre le disponible fourrager et la charge animale, et ils savent que dans les années à venir les éleveurs n'auront que le choix de diminuer le nombre d'animaux pour garder le peu qu'ils seront capables de gérer sur place. « Nous commençons à avoir des difficultés pour alimenter nos animaux et notre propre sécurité lors de la transhumance n'est plus garantie. La situation commence à nous dépasser, nous allons devoir rester chez nous dans les jours à venir pour éviter toutes sortes de problèmes », ont déclaré plus de $60 \%$ des pasteurs transhumants enquêtés à Bougouni.

Cependant, près de $37 \%$ des agriculteurs, $39 \%$ des pasteurs sédentaires et $38 \%$ des pasteurs transhumants interviewés à Koutiala contre seulement et respectivement 22, 18 et $12 \%$ de ces groupes à Bougouni n'ont pas envisagé la possibilité de diminuer significativement les pratiques de la transhumance dans les trente prochaines années. Leur argumentation était basée tout d'abord sur le fait que la transhumance était un mode de vie et non pas seulement un système de production, et qu'il y a toute une société qui vit de ce système de production. Ensuite, ils ont argué que la transhumance était une nécessité qui s’imposait dans les conditions de production animale du Sahel car la bonne productivité des animaux en dépendait. Les mouvements des animaux sont indispensables pour la vie des animaux, par conséquent sans transhumance, pas d'élevage au Sahel : « La transhumance continuera tant que nous vivrons à condition que le gouvernement trouve une alternative pour que nous puissions subvenir aux besoins de nos animaux et à ceux de nos familles ».

\section{DISCUSSION}

Les résultats ont montré que les paysans étaient conscients qu'il y a eu une augmentation des mouvements des animaux au cours des trente dernières années et ils ont observé que la distance parcourue par les animaux lors de la transhumance a eu tendance à s'allonger au fil des années. Des résultats similaires ont été rapportés par Ayantunde et al. (2014), et Schlecht et al. (2001). Les paysans ont constaté que les distances parcourues évoluaient d'une saison à l'autre en fonction des conditions climatiques, de la disponibilité et de la répartition des ressources pastorales (FAO et Cirad, 2012 ; Ayantunde et al., 2011). En effet, suite à la rareté des pâturages et à l'expansion des terres cultivables en zone de pâturages, les transhumants sont obligés de créer des itinéraires alternatifs et de parcourir des centaines de kilomètres pour accéder aux ressources pastorales dans les zones où elles sont disponibles (Bassett et Turner, 2007 ; Ayanda et al., 2013 ; Turner et Hiernaux, 2008). L'implication des agriculteurs dans l'élevage était perçue comme un élément qui a contribué à la réduction de l'accès des groupes transhumants aux ressources pastorales dans le sud du Mali.

De fait, la pratique de l'élevage par les agriculteurs et le manque de fourrage naturel en saison sèche chaude font qu'il y a une collecte 
et un stockage d'une quantité importante des résidus par les agriculteurs juste après la récolte, réduisant ainsi l'accès des pasteurs transhumants aux résidus de récolte (Kiema et al., 2012 ; Sougnabe, 2013). Cette réduction des ressources fourragères et l'augmentation du nombre d'animaux dans les territoires d'accueil sont à l'origine de l'accroissement de la compétition autour des ressources disponibles car chaque acteur défend ses intérêts à tout prix. Cette réalité conduit le plus souvent à des situations tendues et/ou conflictuelles entre les agriculteurs et les éleveurs (Kiema et al., 2014 ; Driel, 1999). De temps en temps des massacres de personnes sont aussi rapportés.

Cependant, l'ampleur des conflits est beaucoup plus élevée dans les zones où la durée de séjour des transhumants est beaucoup plus longue. Par exemple, à Bougouni, on assiste au cours de ces dernières années à une recrudescence du nombre de conflits entre les agriculteurs et les éleveurs (Umutoni, 2016), suite à l'augmentation du nombre de transhumants arrivant dans cette zone et à la durée de leur séjour (Moritz, 2010). En effet, la zone de Bougouni est devenue en partie un lieu de destination pour beaucoup de transhumants en raison des ressources pastorales qu'elle offre. De plus, l'accroissement du nombre de transhumants et de la durée de leur séjour dans cette zone s'explique par la crise socio-politique ivoirienne mentionnée plus haut.

Par ailleurs, dans les zones où la durée de séjour des transhumants s'est raccourcie, le nombre de conflits a considérablement diminué. C'est le cas à Koutiala qui est devenue plutôt une zone de transit pour les transhumants. La cause de cette tendance était la faible disponibilité en pâturages. La plupart des zones qui étaient autrefois destinées aux activités pastorales ont été transformées en zones de culture suite à une démographie en forte hausse. De plus, les points d'abreuvement sont insuffisants. Tous ces facteurs réunis constituaient des obstacles suffisants pour que les transhumants réduisent leur temps de séjour dans cette zone.

Les résultats montrent que les paysans continuent d'observer qu'un grand nombre de troupeaux transhumants vient du Sahel mais ils remarquent aujourd'hui l'arrivée de troupeaux venant d'horizons divers, là où la rareté des pâturages commence à se faire sentir. En conséquence, la transhumance n'est plus une pratique exclusive des Peuls. Cette diversité ethnique dans les pratiques de la transhumance a été rapportée par Banoin et Jouve (2000) au Niger. Une hypothèse expliquant cette évolution est le transfert important au cours des deux dernières décennies du cheptel des éleveurs vers les agriculteurs. On assiste également à une réduction du confiage des animaux à des spécialistes (peuls) puisque les agriculteurs disent ne plus faire confiance aux éleveurs. Tous ces éléments ont conduit à une surcharge animale chez la population bénéficiaire du transfert qui est alors obligée de chercher des pâturages disponibles dans les zones marginales (Banoin et Jouve, 2000), d'où la pratique de la transhumance chez les populations sédentaires.

Cette diversité des groupes ethniques dans la pratique de la transhumance est un signe révélateur d'une augmentation des pratiques de la transhumance. Cette recrudescence des mouvements des animaux pourrait être attribuée à l'augmentation considérable du bétail et à une réduction considérable des ressources pastorales. L'association de ces deux éléments rend difficile la gestion des animaux sur place et oblige les éleveurs à se déplacer pour limiter les risques. Néanmoins, une diminution des pratiques de la transhumance a été rapportée par certains répondants, associant cela à la rareté des pâturages qui obligeait certains éleveurs à déstocker leurs animaux et garder le peu qu'ils étaient capable de gérer sur place. Un autre élément cité à l'origine de cette diminution était les conflits qui ont opposé certains éleveurs avec les communautés d'accueil, poussant certains à abandonner la pratique de la transhumance.

Par ailleurs, les difficultés associées aux pratiques de la transhumance, qui ont de plus bouleversé la société agropastorale, compromettent l'avenir de la transhumance dans le sud du Mali. Globalement, les réflexions sur l'hypothèse d'une réduction significative de la transhumance dans les trente prochaines années ont différé selon les zones d'études. Les zones qui expérimentent une recrudescence des mouvements des animaux ont tendance à adhérer à cette hypothèse. Nous pensons que la recrudescence des conflits dans les zones à forte présence d'éleveurs transhumants au cours des trente dernières années et les difficultés associées aux pratiques de la transhumance, comme la diminution de l'accès aux ressources pastorales pour les éleveurs transhumants et la gestion des animaux transhumants qui devient de plus en plus difficile dans les territoires d'accueil, expliquent la contradiction entre l'augmentation de la transhumance, constatée au cours des trente dernières années par les paysans et leur prévision de sa diminution dans les années à venir.

Au cours des deux dernières décennies, l'avenir des systèmes pastoraux a suscité l'intérêt des experts à cause des sécheresses de plus en plus sévères, la croissance démographique et la multiplication des conflits avec les agriculteurs mettant en péril l'avenir de l'élevage pastoral au Sahel (Niamir-Fuller et Turner, 1999 ; Marty et al., 2006 ; Turner et al., 2014). Turner et al. (2014) affirment que la mobilité des animaux est une stratégie d'adaptation à long terme aux variabilités climatiques et qu'elle continuera de jouer un rôle très important comme stratégie de subsistance chez les pauvres en milieu rural. D'après ces auteurs, la transhumance est une stratégie pour réduire la vulnérabilité des communautés rurales face aux changements climatiques et aussi une stratégie pour limiter l'effet écologique négatif du pâturage du bétail sur les pâturages ouverts. Marty et al. (2006) ont argumenté en disant que les modes individuels de la mobilité vont changer, la mobilité va s'adapter à de nouveaux contextes, à de nouvelles opportunités et contraintes, mais la mobilité va persister et peut être même augmenter. En y regardant de plus près et vu l'importance de l'élevage en milieu rural, il est évident que la rareté des pâturages va conduire à une augmentation de la mobilité du bétail à moins qu'il y ait suffisamment de ressources pour acheter les aliments pour les animaux. De plus d'après une étude, si la transhumance disparaissait, le nombre d'animaux serait réduit de moitié dans vingt ans sans augmentation de la productivité (SWAC/OECD, 2007), ce qui compromettrait encore davantage la sécurité alimentaire dans le monde rural, mais plus particulièrement celle des ménages qui dépendent presque exclusivement de l'élevage.

\section{- CONCLUSION}

Au Mali la pratique de la transhumance a subi des changements importants au cours des trois dernières décennies avec une augmentation des mouvements des animaux. Aujourd'hui, plusieurs groupes ethniques pratiquent la transhumance, contrairement au passé où elle était une spécialité des Peuls.

Les résultats montrent également une recrudescence des conflits entre les agriculteurs et les éleveurs au cours de ces dernières années de sorte que les communautés d'accueil ou de transit des transhumants commencent à être réticentes face à cette pratique. En dépit de cette réticence, certains paysans continuent à soutenir les pratiques de la transhumance en arguant que non seulement c'est un mode de vie mais c'est aussi le seul moyen pour nourrir un grand nombre d'animaux dans les conditions actuelles. Cependant, d'autres paysans ne soutiennent plus ce mode d'élevage car ils le considèrent comme non adapté aux réalités actuelles.

Sur la base de nos résultats, il n'est pas possible de conclure sur la probabilité de disparition ou non de la transhumance dans les trente prochaines années. Néanmoins, l'étude souligne la possibilité d'une réduction des pratiques de la transhumance dans les années à venir. 


\section{Remerciements}

Les auteurs expriment leur gratitude à l'Association malienne d'éveil pour le développement durable (Amedd) pour leur soutien. Ils expriment également leur sincère reconnaissance à la United States Agency for International Development (USAID) pour avoir financé cette étude. Cette recherche a également bénéficié du soutien du programme de recherche du Groupe consultatif pour la recherche agricole internationale (Cgiar) dans les systèmes arides.

\section{REFERENCES}

Ayanda I.F., Oyeyinka R.A., Salau S.A., Ojo F., 2013. Perceived effects of climate change on transhumance pastoralists in Ogun State, Nigeria. J. Agric. Sci., 5: 190-198, doi: 10.5539/jas.v5n4p190

Ayantunde A.A., Asse R., Said M.Y., Fall A., 2014. Transhumant pastoralism, sustainable management of natural resources and endemic ruminant livestock in the sub-humid zone of West Africa. Environ. Dev. Sustain., 16: 1097-1117, doi: 10.1007/s10668-014-9515-z

Ayantunde A.A., De Leeuw J., Turner M.D., Said M., 2011. Challenges of assessing the sustainability of (agro) pastoral systems. Livest. Sci., 139: 30-43, doi: 10.1016/j.livsci.2011.03.019

Ayantunde A.A., Williams T.O., Udo H.M.J., Fernández-Rivera S. Hiernaux P., Van Keulen H., 2000. Herders' perceptions, practice, and problems of night grazing in the Sahel: Case studies from Niger. Hum. Ecol., 28: 109-130, doi: 10.1023/A:1007031805986

Banoin M., Gueye C., Soumana I., Ali M., Jouve P., 1996. Péjorations climatiques et évolution des pratiques de transhumance en zone agropastorale sahélienne : cas de l'arrondissement de Mayahi, au Niger. In : Gestion des terroirs et des ressources naturelles au Sahel (éd. Jouve P.). Cirad-SAR, Cnearc, Montpellier, France, 43-60

Banoin M., Jouve P., 2000. Déterminants des pratiques de transhumance en zone agro pastorale sahélienne : cas de l'arrondissement de Mayahi, au Niger. In : Rupture : nouveaux enjeux, nouvelles fonctions, nouvelle image de l'élevage sur parcours (éds Bourbouze A., Qarro M.). Ciheam, Montpellier, France, 91-105 (Options Méditerr. Sér. A. Séminaires méditerranéens; $n^{\circ} 39$ )

Bassett T.J., Turner M., 2007. Sudden shift or migratory drift? Fulbe herd movements to the Sudano-Guinean region of West Africa. Hum. Ecol., 35: 33-49, doi: 10.1007/s10745-006 9067-4

Creswell J.W., 2014. Research design: Qualitative, quantitative and mixed methods approaches. Sage Publication, California, USA, 273 p.

Dembele C., 2008. Sécuriser le pastoralisme en Afrique de l'Est et de I'Ouest. Protéger et promouvoir la mobilité du bétail. IIED / Sahel Eco, Mali

Driel A.V., 1999. The end of the herding contract: Decreasing complementary linkage between Fulbe pastoralists and Dendi agriculturalists in Northern Benin. In: Pastoralists under pressure? Fulbe societies confronting change in West Africa (Eds. Azarya V., Breedveld A., Bruijn M.D., Dijk H.V.). Brill, Leiden, Netherlands, 191-209 (Social, economic and political studies of the Middle East and Asia)

Duggleby W., 2005. What about focus group interaction data. Qual. Health Res., 15: 832 840, doi: 10.1177/1049732304273916
FAO, Cirad, 2012. Atlas des évolutions des systèmes pastoraux au Sahel 1970-2012. FAO, Rome, Italie

ILRI, 2010. Sustainable management of globally significant endemic ruminant livestock in West Africa (PROGEBE). Gambia Baseline Report. International Livestock Research Institute, Nairobi, Kenya

Kiema A., Sawadogo I., Ouedraogo T., Nianogo A.J., 2012. Stratégies d'exploitation du fourrage par les éleveurs de la zone sahélienne du Burkina Faso. Int. J. Biol. Chem. Sci., 6: 1492-1505, doi: 10.4314/ijbcs. v6i4.8

Kiema A., Tontibomma G.B., Zampaligre N., 2014. Transhumance et gestion des ressources naturelles au Sahel : contraintes et perspectives face aux mutations des systèmes de productions pastorales. VertigO, 14 (3), doi : 10.4000/vertigo.15404

Marty A., Bonnet B., Guibert B., 2006. La mobilité pastorale et sa viabilité. Entre atouts et défis. Institut de recherches et d'applications des méthodes de développement, Paris, France

Moritz M., 2010. Understanding herder-farmer conflicts in West Africa: Outline of a processual approach. Hum. Organ., 69: 138-148, doi: 10.17730/humo.69.2.aq85k02453w83363

Moritz M., Kyle B., Nolan K., Patrick S., Shaffer M., Thampy G., 2009. Too many people and too few livestock in West Africa? An evaluation of Sandford's thesis. J. Dev. Stud., 45: 1113-1133, doi: 10.1080/00220380902811058

Niamir-Fuller M., Turner M.D., 1999. A review of recent literature on pastoralism and transhumance in Africa. In: Managing mobility in African rangelands: the legitimization of transhumance (Ed. NiamirFuller M.). IT Publications, Exeter, UK, 18-46

Schlecht E., Hiernaux P., Turner D.M., 2001. Mobilité régionale du bétail, nécessité et alternatives ? In : Elevage et gestion de parcours au Sahel, implication pour le développement (éds. Tielkes E., Schlecht E, Hiernaux P.). Verlag Ulrich, Stuttgart, Allemagne, 65-77

Sougnabe P., 2013. La sédentarisation comme moyen d'adaptation aux baisses de la pluviométrie chez les éleveurs peuls en savane tchadienne. VertigO, 13 (1), doi : 10.4000/vertigo.13468

SWAC/OECD, 2007. Promoting and supporting change in transhumant pastoralism in the Sahel and West Africa. SWAC/OECD, Paris, France. (Policy Note ; 3 )

Turner M.D., 2000. Drought, domestic budgeting and wealth distribution in Sahelian households. Dev. Change, 31, doi: 10.1111/14677660.00187

Turner M.D., Hiernaux P., 2008. Changing access to labor, pastures, and knowledge: The extensification of grazing management in SudanoSahelian West Africa. Hum. Ecol., 26: 59-80, doi: 10.1007/s10745007-9149-y

Turner D.M., McPeak J.G., Ayantunde A., 2014. The role of livestock mobility in the livelihood strategies of rural peoples in semi-arid West Africa. Hum. Ecol., 42: 231-247, doi: 10.1007/s10745-013-9636-2

Umutoni C., 2016. Analyse des modes de gestion des ressources naturelles dans le cadre d'identification des facteurs d'amélioration de la productivité du bétail dans les systèmes mixtes agriculture-élevage en zone soudano-sahélienne du Mali. Thèse Doct., Université Cheikh Anta Diop, Dakar, Sénégal, 142 p. 


\section{Summary}

Umutoni C., Ayantunde A.A., Sawadogo G.J. Local knowledge of transhumance practices in the Sudano-Sahelian zone of Mali

Studies on transhumance have often focused on pastoralists in particular the Fulanis - of Sahelian areas. To give another perspective, the present study investigated transhumance practices in the Sudano-Sahelian zone of Mali by integrating the perception of farmers and sedentary herders affected by transhumance in the host areas. Possible scenarios of transhumant pastoralism for the future were also analyzed. Data were collected through focus groups and individual interviews. The results showed that transhumance was no longer solely practiced by Fulani pastoralists but involved various ethnic groups. Over $70 \%$ of the interviewed farmers, sedentary pastoralists and transhumant pastoralists in the two study sites reported that the benefits of transhumance for the host communities in terms of manure deposit in crop fields had been decreasing. Although the majority of respondents reported an increase in transhumance practices in the last thirty years, they forecast their decline in the coming years. The increasing competition over grazing resources between members of host communities and transhumant pastoralists in Southern Mali creates a deadlock and frustrations expressed by mutual distrust.

Keywords: transhumance, local community, agropastoral system, land conflict, animal husbandry, Mali

\section{Resumen}

Umutoni C., Ayantunde A.A., Sawadogo G.J. Conocimiento local de las prácticas de trashumancia en la zona sudanosaheliana de Malí

Los estudios sobre las prácticas de trashumancia se han a menudo enfocado en pastores - en particular los Peul - de las áreas sahelianas. Para dar otra perspectiva, el presente estudio investigó las prácticas trashumancia en la zona sudanosaheliana de Malí, integrando la percepción de los finqueros y pastores sedentarios afectados por la trashumancia in las zonas de acogida. También se analizaron posibles escenarios de pastoreo trashumante para el futuro. Los datos se colectaron a través de grupos de enfoque y entrevistas individuales. Los resultados muestran que las trashumancia no era ya únicamente practicada por los pastores peul pero que involucraba varios grupos étnicos. Más de $70 \%$ de los finqueros entrevistados, pastores sedentarios y pastores trashumantes en los dos sitios de estudio reportaron que los beneficios de la trashumancia para las comunidades de acogida en términos de deposito de estiércol en los campos de cultivo estaban disminuyendo. Aunque la mayoría de los entrevistados reportaron un aumento en la des prácticas trashumancia en los últimos treinta años, ellos prevén una disminución en los años que vienen. La competencia creciente sobre los recursos de pastoreo entre miembros de las comunidades de acogida y los pastores trashumantes en el sur de Malí crea parálisis y frustraciones expresadas por una desconfianza mutua.

Palabras clave: trashumancia, communidad local, sistema agropascícola, conflicto por la tierra, ganadería, Malí 
